\title{
Photodynamic therapy using mannose-conjugated chlorin e6 stimulates tumor immunity by increasing cell surface calreticulin in cancer cells and promoting macrophage phagocytosis
}

\section{Yuka Kimura}

Nagoya City University: Nagoya Shiritsu Daigaku

Hiromasa Aoki

Nagoya City University: Nagoya Shiritsu Daigaku

Tatsuki Soyama

Nagoya City University: Nagoya Shiritsu Daigaku

Akira Sakuragi

Nagoya City University: Nagoya Shiritsu Daigaku

\section{Yuto Otsuka}

Nagoya City University: Nagoya Shiritsu Daigaku

Akihiro Nomoto

Osaka Prefecture University

\section{Shigenobu Yano}

Nara Women's University

\section{Hirotada Nishie}

Nagoya City University Graduate School of Medical Sciences and Medical School: Nagoya Shiritsu Daigaku Daigakuin Igaku Kenkyuka Igakubu

Hiromi Kataoka

Nagoya City University Graduate School of Medical Sciences and Medical School: Nagoya Shiritsu Daigaku Daigakuin Igaku Kenkyuka Igakubu

Mineyoshi Aoyama ( $\square$ aomine@phar.nagoya-cu.ac.jp)

Nagoya City University Graduate School of Pharmaceutical Sciences https://orcid.org/0000-00029976-6465

\section{Short Report}

Keywords: cells, chlorin, cancer, tumor, macrophages

Posted Date: October 19th, 2021 
DOl: https://doi.org/10.21203/rs.3.rs-974706/v1

License: (c) (1) This work is licensed under a Creative Commons Attribution 4.0 International License. Read Full License 


\section{Abstract}

Photodynamic therapy (PDT) damages cancer cells via photosensitization using harmless laser irradiation. We synthesized a new photosensitizer, mannose-conjugated-chlorin e6 (M-chlorin e6), which targets mannose receptors that are highly expressed on M2-like tumor-associated macrophages (M2TAMs) and cancer cells. In our previous study, we demonstrated that M-chlorin e6 PDT reduces tumor volume and decreases the proportion of M2-TAMs. Whether M-chlorin e6 PDT-treated cancer cells activate tumor immunity remains unclear, although the decrease in M2-TAMs is thought to be a direct injurious effect of M-chlorin e6 PDT. Calreticulin (CRT) is exposed the surface of the membrane of cancer cells in response to treatment with chemotherapeutic agents such as anthracycline and oxaliplatin. Surface-exposed CRT induces phagocytosis of CRT receptor-positive cells, including macrophages, resulting in antigen processing and the induction of T cell-mediated anticancer immune responses. In the present study, we found that M-chlorin e6 PDT increases CRT on the surface of cancer cells, leading to macrophage phagocytosis of cancer cells. Furthermore, M-chlorin e6 PDT increases $\mathrm{CD} 80^{+} \mathrm{CD}_{86}{ }^{+}$macrophages. These results suggest that M-chlorin e6 PDT stimulates T cell-mediated tumor immunity by enhancing the macrophage phagocytosis of cancer cells and the antigen-presenting capability of macrophages.

\section{Introduction}

Photodynamic therapy (PDT) is a new cancer treatment that kills target cells using non-damaging visible laser irradiation and a nontoxic photosensitizer [1]. When a photosensitizer is irradiated with a laser at a specific wavelength, the photosensitizer gains energy and enters an excited state. When the photosensitizer returns to the ground state, it releases reactive oxygen species, which induces the target cell to undergo apoptosis. This mechanism allows the laser to cause damage only to the irradiated tumor site. Therefore, PDT is expected to be a less invasive, site-selective approach for treating cancer and a variety of other diseases.

In Japan, the photosensitizing agents porfimer sodium (also known as Photofrin) and taraporphine sodium (also known as Laserphyrin) have been approved for clinical use. However, PDT with porfimer sodium has several disadvantages, such as a high frequency of skin phototoxicity and the need for patients to avoid direct sunlight for a long period time, whereas talaporfin sodium has low tumor selectivity [1-3]. During our photosensitizer research [4-6], we generated a new photosensitizer, mannose-conjugated chlorin e6 (M-chlorin e6), which overcomes the drawbacks of existing photosensitizers [7]. M2-like tumor-associated macrophages (M2-TAMs) highly express mannose receptors (e.g., CD206). Therefore, M-chlorin e6 is targeted to M2-TAMs, which produce anti-inflammatory cytokines such as IL-10 and TGF- $\beta$ and promote immunosuppression, tumor growth, and tumor development [8]. Cancer cells also take up M-chlorin e6 (recognized as mannose-conjugated chlorin) via a variety of pathways, including glucose transporter 1 . In our previous study using syngeneic tumor model mice, we demonstrated that M-chlorin e6 PDT reduces M2-TAMs and damages cancer cells, thus suppressing tumor growth [7]. 
Immunogenic cell death (ICD) is a type of cancer cell death caused by certain therapies, such as chemotherapy and radiotherapy. ICD activates tumor-specific immune responses in response to damageassociated molecular patterns (DAMPs) released from dying tumor cells [9]. Calreticulin (CRT), a protein belonging to the DAMP family, is the most abundant calcium-binding chaperone in the lumen of the endoplasmic reticulum [10]. CRT is exposed at the surface of the cell membrane in response to treatment with ICD inducers such as anthracycline and oxaliplatin. Surface-exposed CRT acts as a de novo uptake signal for phagocytes expressing the CRT receptor (e.g., $\mathrm{CD} 91^{+}$cells such as macrophages), resulting in enhanced recognition of stressed or dying cells [10]. Macrophages, which detect binding of CRT to CD91 ${ }^{+}$, phagocytose tumor cells and initiate antigen processing and presentation to T cells [11]. Thus, CRT exposure is an important process of ICD that induces T cell-mediated anticancer immune responses. In this study, we attempted to demonstrate a new application of M-chlorin e6 PDT as an ICD inducer by analyzing CRT exposure and phagocytosis by macrophages.

\section{Materials And Methods}

\section{Photosensitizers}

M-chlorin e6 (methyl(7S,8S)-18-ethyl-5-(2-methoxy-2-oxoethyl)-7-(3-methoxy-3-oxopropyl)-2,8,12,17tetramethyl-13-(1-(3-((2S,3S,4S,5S,6R)-3,4,5-trihydroxy-6-(hydroxymethyl)tetrahydro-2H-pyran-2yl)thio)propoxy)ethyl)-7H,8H-porphyrin-3-carboxylate) and G-chlorin e6 (methyl(7S,8S)-18-ethyl-5-(2methoxy-2-oxoethyl)-7-(3-methoxy-3-oxopropyl)-2,8,12,17-tetramethyl-13-(1-(3-(((2S,3R,4S,5S,6R)-3,4,5trihydroxy-6-(hydroxymethyl)tetrahydro-2H-pyran-2-yl)thio)propoxy)ethyl)-7H,8H-porphyrin-3-carboxylate) were synthesized and provided by the laboratory of Osaka Prefecture University (Osaka, Japan). Mchlorin e6 was synthesized as described previously [7].

\section{Cell culture}

CT26 mouse colon cancer cells were purchased from the American Type Culture Collection (ATCC, Manassas, VA, USA) and cultured at $37^{\circ} \mathrm{C}$ in a $5 \% \mathrm{CO}_{2}, 95 \%$ air environment. CT26 cells were grown in low-glucose (1000 mg/L) Dulbecco's modified Eagle's medium (DMEM, Wako, Osaka, Japan) supplemented with $10 \%$ fetal bovine serum (FBS), $100 \mathrm{U} / \mathrm{mL}$ penicillin, and $100 \mu \mathrm{g} / \mathrm{mL}$ streptomycin. RAW264.7 mouse macrophages were purchased from the ATCC and cultured at $37^{\circ} \mathrm{C}$ in a $5 \% \mathrm{CO}_{2}, 95 \%$ air environment. RAW264.7 cells were grown in (1000 mg/L) RPMI-1640 (Wako) medium supplemented with $10 \% \mathrm{FBS}, 100 \mathrm{U} / \mathrm{mL}$ penicillin, and $100 \mu \mathrm{g} / \mathrm{mL}$ streptomycin.

\section{Flow cytometric analysis of CRT}

CT26 cells $\left(n=1 \times 10^{6}\right)$ were seeded in 10 -cm dishes and incubated for $24 \mathrm{~h}$ at $37^{\circ} \mathrm{C}$ with $5 \% \mathrm{CO}_{2}$ and divided into a control group and PDT group. In the control group, the medium was changed to fresh DMEM low glucose for another $24 \mathrm{~h}$. In the PDT group, the medium was changed to fresh DMEM low glucose containing M-chlorin e6 (120 nM) and incubated for another $24 \mathrm{~h}$, after which the medium was 
replaced with phosphate-buffered saline (PBS), and the cells were irradiated with LED light $(660 \mathrm{~nm}, 16$ $\mathrm{J} / \mathrm{cm}^{2}$ ) using an LEDR-660DL (Opto Code, Tokyo, Japan). In both groups, the medium was then changed again to fresh DMEM low glucose (without M-chlorin e6) and incubated for $1 \mathrm{~h}$, after which the cells were detached using TrypLE ${ }^{T M}$ Express Enzyme (1x) without phenol red (Thermo Fisher Scientific, San Diego, CA, USA). The cells were counted and then aliquoted at $1 \times 10^{6}$ cells per sample, after which primary antibody (Calreticulin [D3E6] XP ${ }^{\circledR}$ Rabbit mAb; Cell Signaling Technology, Danvers, MA, USA) was added, and the cells were placed on ice for $30 \mathrm{~min}$. The secondary antibody (Alexa Fluor ${ }^{\mathrm{TM}} 488 \mathrm{~F}[\mathrm{ab}$ ']2 fragment of goat anti-rabbit IgG [H+L]; Invitrogen, Waltham, MA, USA) was then added, and the cells were kept on ice for 30 min. The cells were then washed with PBS, suspended in Stain Buffer (BD Biosciences, San Jose, $C A, U S A$ ), and 7-AAD (BD Biosciences) was added. Finally, the cells were analyzed using a FACS Verse $^{\mathrm{TM}}$ flow cytometer (BD Biosciences).

\section{Phagocytosis assay}

CT26 cells $\left(n=3 \times 10^{5}\right)$ were seeded in 3.5 -cm glass-bottom dishes and incubated for $24 \mathrm{~h}$ at $37^{\circ} \mathrm{C}$ with $5 \%$ $\mathrm{CO}_{2}$ and then divided into a control group and PDT group. In the control group, the medium was changed to fresh DMEM low glucose containing M-chlorin e6 (120 nM), and the cells were incubated for another $24 \mathrm{~h}$. In the PDT group, the medium was changed to fresh DMEM low glucose containing M-chlorin e6 $(120 \mathrm{nM})$, and the cells were incubated for another $24 \mathrm{~h}$, after which the medium was replaced with PBS, and the cells were irradiated with LED light $\left(660 \mathrm{~nm}, 16 \mathrm{~J} / \mathrm{cm}^{2}\right)$ using an LEDR-660DL. In both groups, the medium was changed again to DMEM low glucose (without M-chlorin e6), and the cells were incubated for $1 \mathrm{~h}$, after which the medium was changed to RPMI-1640 medium containing suspended RAW264.7 cells $\left(n=1 \times 10^{5}\right)$ labeled with BCECF-AM special packaging (Dojindo, Kumamoto, Japan), and the cells were incubated for 3-24 h. Phagocytosis was evaluated using an LSM800 confocal microscope (Carl Zeiss, Oberkochen, Germany).

\section{Flow cytometric analysis of CD80 and CD86}

CT26 cells $\left(n=1 \times 10^{6}\right)$ were seeded in $10-\mathrm{cm}$ dishes and cultured for $24 \mathrm{~h}$ at $37^{\circ} \mathrm{C}$ with $5 \% \mathrm{CO}_{2}$ and then divided into a control group and PDT group. In the control group, the medium was changed to fresh DMEM low glucose, and the cells were incubated for another $24 \mathrm{~h}$. In the PDT group, the medium was changed to fresh DMEM low glucose containing M-chlorin e6 $(120 \mathrm{nM})$, and the cells were incubated for another $24 \mathrm{~h}$, after which the medium was replaced with PBS, and the cells were irradiated with LED light $\left(660 \mathrm{~nm}, 16 \mathrm{~J} / \mathrm{cm}^{2}\right)$ using an LEDR-660DL. In both groups, the medium was again changed to DMEM low glucose (without M-chlorin e6), and the cells were incubated for $1 \mathrm{~h}$, after which the medium was changed to RPMI-1640 containing suspended RAW264.7 cells $\left(n=1 \times 10^{6}\right)$, and the cells were incubated for another $24 \mathrm{~h}$. The cells were then detached using TrypLE ${ }^{\mathrm{TM}}$ Express Enzyme (1x) without phenol red and counted. The cells were then aliquoted at $1 \times 10^{6}$ cells per sample, Mouse BD Fc block ${ }^{\mathrm{TM}}$ was added, and the cells were placed on ice for 10 min. Finally, antibody (PE hamster anti-mouse CD80, PE-Cy7 rat antimouse CD86 [BD Biosciences]) or isotype control (PE hamster IgG2 kappa isotype control, PE-Cy 7 rat 
IgG2a kappa isotype control [BD Biosciences]) was added, and the cells were kept on ice for $30 \mathrm{~min}$. The cells were washed with PBS, suspended in Stain Buffer, and 7-AAD was added. The cells were analyzed using a FACS Verse ${ }^{\mathrm{TM}}$ flow cytometer (BD Biosciences).

\section{Statistical analyses}

All statistical analyses were performed using EZR software (Saitama Medical Center, Jichi Medical University, Saitama, Japan), which is a graphical user interface for R. More precisely, EZR is a modified version of $\mathrm{R}$ Commander designed to add statistical functions frequently used in biostatistics [12]. The two-tailed non-paired Student's $t$-test was used for comparisons between two groups. Data are presented as the mean \pm standard error. A $p$ value of $<0.05$ was considered statistically significant.

\section{Results}

M-chlorin e6 PDT increases cell surface CRT in cancer cells and activates phagocytosis of cancer cells by macrophages

Cell surface CRT in cancer cells treated with M-chlorin e6 PDT was analyzed by flow cytometry. CRT intensity was significantly increased in the PDT group compared with the control group (Fig. 1A and 1B). To examine whether M-chlorin e6 PDT promotes phagocytosis, we co-cultured M chlorin e6 PDT-treated CT26 cells with RAW264.7 cells. The proportion of phagocytosed cells was significantly higher in the PDT group compared with the control after $24 \mathrm{~h}$ of co-culture (Fig. 1C and 1D).

\section{M-chlorin E6 Pdt Increases The Proportion Of Cd80+cd86+ Macrophages}

We also analyzed the proportion of $C D 80^{+} \mathrm{CD} 86^{+}$RAW264.7 cells using flow cytometry after $24 \mathrm{~h}$ of coculture. The proportion of $\mathrm{CD} 80^{+} \mathrm{CD} 86^{+}$cells was significantly higher in the PDT group compared with the control group (Fig. 2A and 2B). In our previous study [7], we showed that M-chlorin e6 PDT reduces the percentage of M2-TAMs and increases that of anti-tumor M1-like TAMs (M1-TAMs) in tumor tissues, and directly damages cancer cells, resulting in a decrease in tumor volume (Fig. $2 \mathrm{C}$ ). The present experiments suggest that M-chlorin e6 PDT increases CRT on the surface of tumor cells, induces the phagocytosis of these cells by macrophages, and enhances antigen presentation by increasing the proportion of CD $80^{+} \mathrm{CD} 86^{+}$macrophages (Fig. 2D).

\section{Discussion}

Some types of PDT reportedly enhance tumor immunity via induction of ICD [6,13-15], but which type of photosensitive PDT triggers such an event remains unclear. M-chlorin e6 has the potential for clinical application as a photosensitizer because of its high tumor selectivity, rapid elimination from the body, direct tumor damaging activity, and reduction of M2-TAMs [7]. Our finding of increased CRT expression 
on the surface of cancer cells represents a new characteristic of M-chlorin e6. The precise mechanism by which CRT is exposed on the cell surface by M-chlorin e6 PDT needs to be clarified in future studies. The increased phagocytosis of cancer cells by macrophages might be related to the elevation of cell surface CRT expression [11]. However, the possibility that other factors, such as other types of DAMPs, may also be involved must be taken into account and therefore represents a subject for further investigation.

CD80 and CD86 are major proteins required for antigen presentation to T cells, and their expression on macrophages and dendritic cells plays an important role in tumor immunity [16]. For example, when cisplatin was administered to a control group of tumor model mice, $50 \%$ of the mice survived. However, when $\mathrm{CD} 80^{-} \mathrm{CD} 86^{-}$tumor model mice were treated with cisplatin, the majority of mice did not survive, even with the suppression of tumor growth [17]. These results suggest that CD80/CD86 play an extremely important role in tumor immunity. These results also indicate that certain chemotherapeutic agents, such as cisplatin, enhance CD80/CD86-mediated tumor immunity and that CD80/CD86 play pivotal roles in tumor immunity. In this study, treatment of cancer cells using M-chlorin e6 PDT resulted in an increase in the number of $\mathrm{CD} 80^{+} \mathrm{CD} 86^{+}$macrophages. This result suggests that M-chlorin e6 not only promotes phagocytosis of cancer cells but also enhances tumor immunity by activating antigen presentation by macrophages. Presumably, exposure of CRT and other DAMP proteins or the release of various cytokines by PDT-treated cancer cells is involved in the increased expression of CD80/CD86 by macrophages [18]. CD80/CD86 are known markers of tumor-suppressive M1-type macrophages [19], and these results may explain the increase in M1-TAMs after PDT treatment in the in vivo tumor model in the previous study. As the present study focused only on macrophages, future studies will examine whether M-chlorin e6 PDT also activates dendritic cells.

In conclusion, M-chlorin e6 PDT increases expression of cell surface CRT by cancer cells and activates macrophage phagocytosis of CRT-expressing cancer cells. M-chlorin e6 PDT also increases the proportion of $\mathrm{CD} 80^{+} \mathrm{CD} 86^{+}$macrophages. These results suggest that M-chlorin e6 PDT stimulates T cellmediated tumor immunity by enhancing both the phagocytosis of cancer cells and antigen presentation by macrophages. The results of this study are expected to bring M-chlorin e6 PDT even closer to clinical application.

\section{Declarations}

\section{Author contributions}

Yuka Kimura: Conceptualization, Methodology, Formal analysis, Investigation, Data curation, Writingoriginal draft, Visualization. Hiromasa Aoki: Conceptualization, Methodology, Formal analysis, Investigation, Data curation, Writing-original draft, Funding acquisition. Tatsuki Soyama: Conceptualization, Formal analysis, Writing-original draft. Akira Sakuragi: Conceptualization, Formal analysis, Writing-original draft. Yuto Otsuka: Conceptualization, Formal analysis, Writing-original draft. Akihiro Nomoto: Conceptualization, Formal analysis, Providing materials, Writing-review \& editing. Shigenobu Yano: Conceptualization, Formal analysis, Providing materials, Writing-review \& editing. 
Hirotada Nishie: Conceptualization, Formal analysis, Writing-review \& editing. Mineyoshi Aoyama: Conceptualization, Formal analysis, Writing-review \& editing, Supervision, Project administration, Funding acquisition. All authors read and approved the final manuscript.

\section{Acknowledgments}

We acknowledge the assistance of the Research Equipment Sharing Center at Nagoya City University. All illustrations were created with Biorender.com. This work was supported in part by Grants-in-Aid for Scientific Research (KAKEN) from the Japan Society for the Promotion of Science (grant numbers 20K22715, 19H02791, 18K05161, 18K15758, 20K08391, and 20K08211) and the Ichihara International Scholarship Foundation.

\section{Statements and Declarations}

The authors declare no competing interests.

\section{References}

1. Ormond AB, Freeman HS. Dye Sensitizers for Photodynamic Therapy. Materials. 2013;6:817-40.

2. Muragaki Y, Akimoto J, Maruyama T, Iseki H, Ikuta S, Nitta M, et al. Phase II clinical study on intraoperative photodynamic therapy with talaporfin sodium and semiconductor laser in patients with malignant brain tumors. J Neurosurg. 2013;119:845-52.

3. Baskaran R, Lee J, Yang S-G. Clinical development of photodynamic agents and therapeutic applications. Biomater Res. 2018;22:25.

4. Nishie $H$, Kataoka $H$, Yano S, Yamaguchi $H$, Nomoto A, Tanaka $M$, et al. Excellent antitumor effects for gastrointestinal cancers using photodynamic therapy with a novel glucose conjugated chlorin e6. Biochem Biophys Res Commun. 2018;496:1204-9.

5. Osaki T, Hibino S, Yokoe I, Yamaguchi H, Nomoto A, Yano S, et al. A Basic Study of Photodynamic Therapy with Glucose-Conjugated Chlorin e6 Using Mammary Carcinoma Xenografts. Cancers. 2019;11:636.

6. Tanaka M, Kataoka H, Yano S, Sawada T, Akashi H, Inoue M, et al. Immunogenic cell death due to a new photodynamic therapy (PDT) with glycoconjugated chlorin (G-chlorin). Oncotarget. 2016;7:47242-51.

7. Soyama T, Sakuragi A, Oishi D, Kimura Y, Aoki H, Nomoto A, et al. Photodynamic therapy exploiting the anti-tumor activity of mannose-conjugated chlorin e6 reduced M2-like tumor-associated macrophages. Transl Oncol. 2021;14:101005.

8. Chen Y, Song Y, Du W, Gong L, Chang H, Zou Z. Tumor-associated macrophages: an accomplice in solid tumor progression. J Biomed Sci. 2019;26:78. 
9. Ahmed A, Tait SWG. Targeting immunogenic cell death in cancer. Mol Oncol. 2020;14:2994-3006.

10. Schcolnik-Cabrera A, Oldak B, Juárez M, Cruz-Rivera M, Flisser A, Mendlovic F. Calreticulin in phagocytosis and cancer: opposite roles in immune response outcomes. Apoptosis. 2019;24:24555.

11. Martins I, Kepp O, Galluzzi L, Senovilla L, Schlemmer F, Adjemian S, et al. Surface-exposed calreticulin in the interaction between dying cells and phagocytes. Ann N Y Acad Sci. 2010;1209:7782.

12. Kanda Y. Investigation of the freely available easy-to-use software "EZR" for medical statistics. Bone Marrow Transplant. 2013;48:452-8.

13. Garg AD, Dudek AM, Ferreira GB, Verfaillie T, Vandenabeele P, Krysko DV, et al. ROS-induced autophagy in cancer cells assists in evasion from determinants of immunogenic cell death. Autophagy. 2013;9:1292-307.

14. Ji J, Fan Z, Zhou F, Wang X, Shi L, Zhang H, et al. Improvement of DC vaccine with ALA-PDT induced immunogenic apoptotic cells for skin squamous cell carcinoma. Oncotarget. 2015;6:17135-46.

15. Turubanova VD, Balalaeva IV, Mishchenko TA, Catanzaro E, Alzeibak R, Peskova NN, et al. Immunogenic cell death induced by a new photodynamic therapy based on photosens and photodithazine. J Immunother Cancer. 2019;7:350.

16. Chikuma S. Basics of PD-1 in self-tolerance, infection, and cancer immunity. Int J Clin Oncol. 2016;21:448-55.

17. Beyranvand Nejad E, van der Sluis TC, van Duikeren S, Yagita H, Janssen GM, van Veelen PA, et al. Tumor Eradication by Cisplatin Is Sustained by CD80/86-Mediated Costimulation of CD8+ T Cells. Cancer Res. 2016;76:6017-29.

18. Rao A, Strauss O, Kokkinou E, Bruchard M, Tripathi KP, Schlums H, et al. Cytokines regulate the antigen-presenting characteristics of human circulating and tissue-resident intestinal ILCs. Nat Commun. 2020;11:1-15.

19. Bertani FR, Mozetic P, Fioramonti M, luliani M, Ribelli G, Pantano F, et al. Classification of M1/M2polarized human macrophages by label-free hyperspectral reflectance confocal microscopy and multivariate analysis. Sci Rep. 2017;7:8965.

\section{Figures}


A

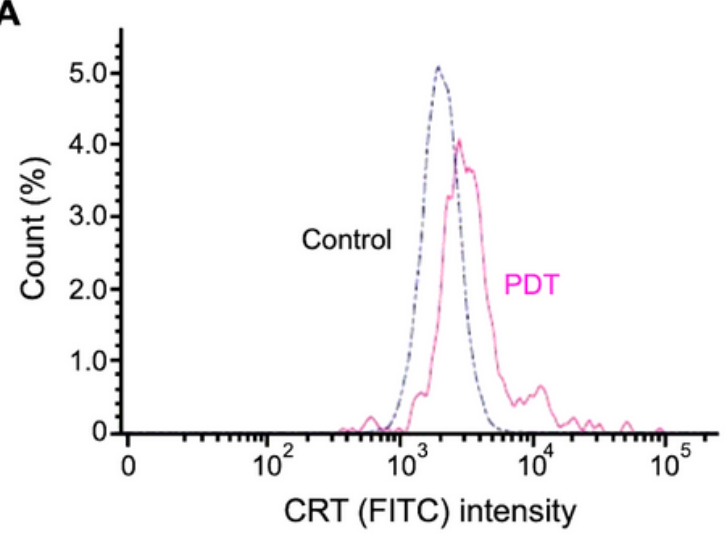

C

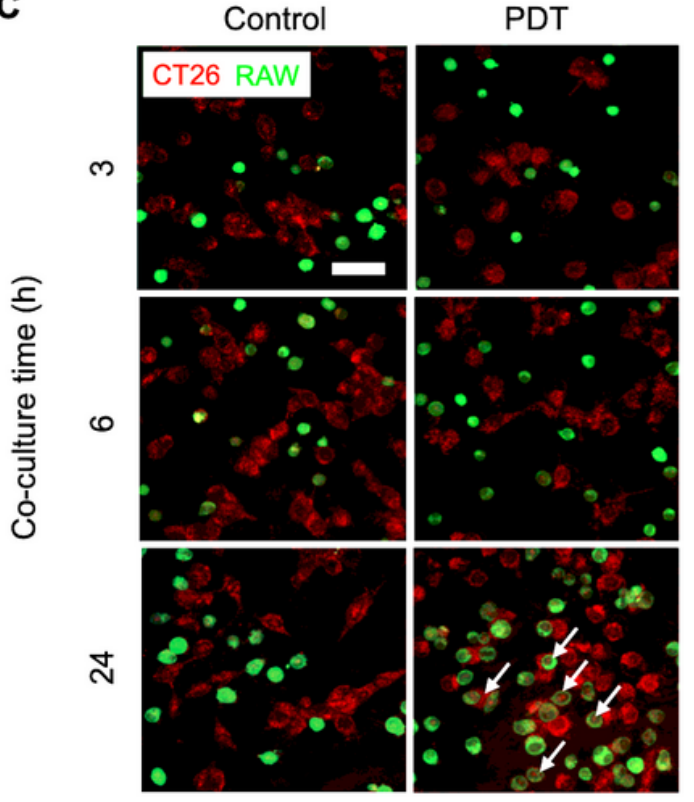

B

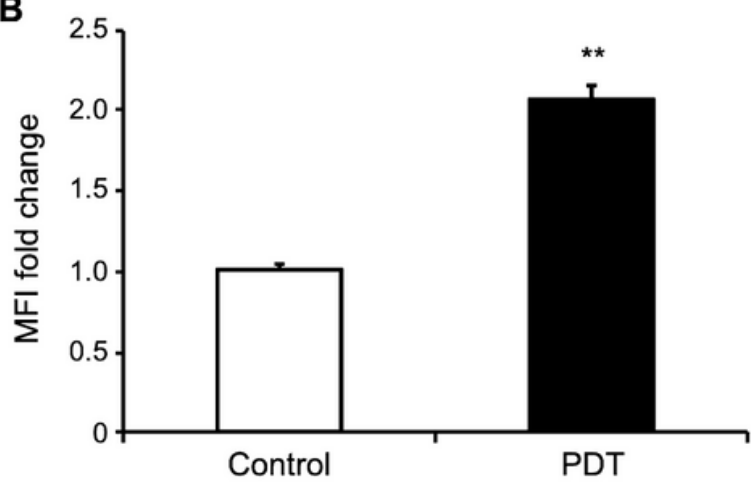

D

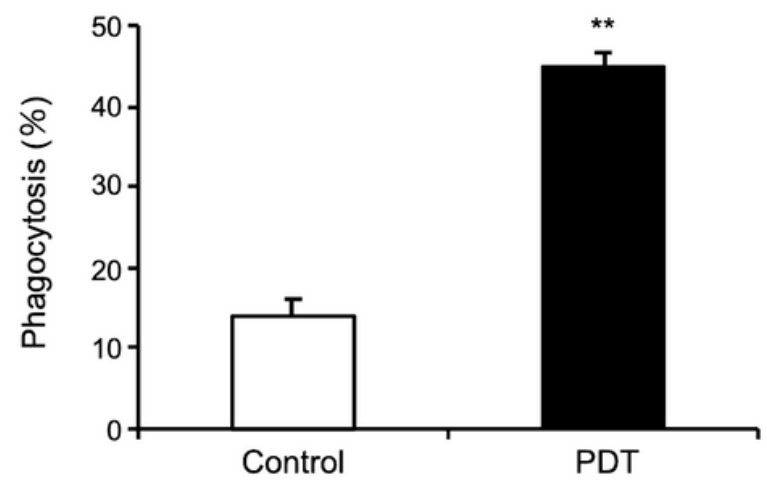

\section{Figure 1}

Effect of M-chlorin e6 PDT on CRT expression on the surface of cancer cells and phagocytosis of cancer cells by macrophages. (A) Representative histogram of flow cytometric analyses after $1 \mathrm{~h}$ of M-chlorin e6 PDT. (B) Relative mean fluorescence intensity (MFI) of CRT. Data are presented as mean $\pm S E(n=3$; ${ }^{*} \mathrm{p}<0.01$; Student's t-test). (C) Representative images of phagocytosis of cancer cells by macrophages after 3 h, 6 h, and 24 h of co-culture with macrophages. M-chlorin e6 (CT26 cells) shown in red. BCECFAM (RAW264.7) shown in green. Scale bars $=50 \mu \mathrm{m}$. (D) The proportion of phagocytosis after $24 \mathrm{~h}$ of coculture with macrophages. Data are presented as mean $\pm S E(n=3 ; * \star p<0.01$; Student's t-test). 
A
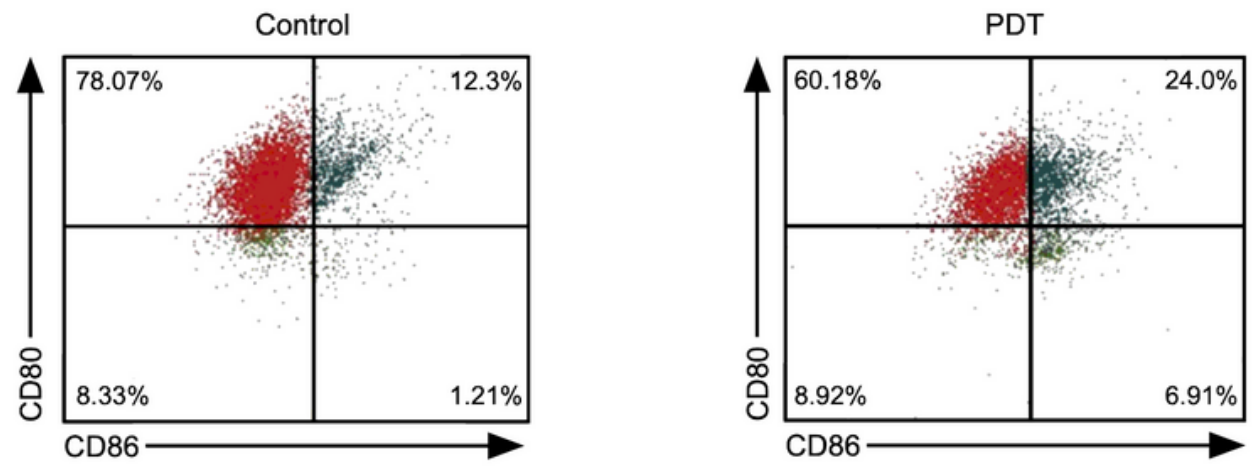

B

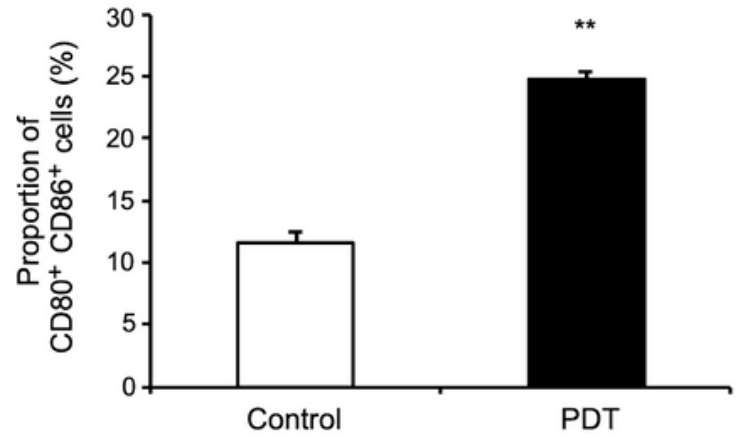

C

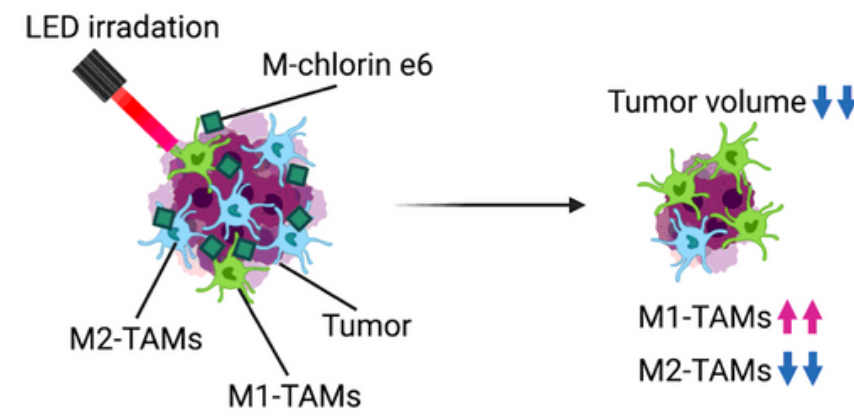

D

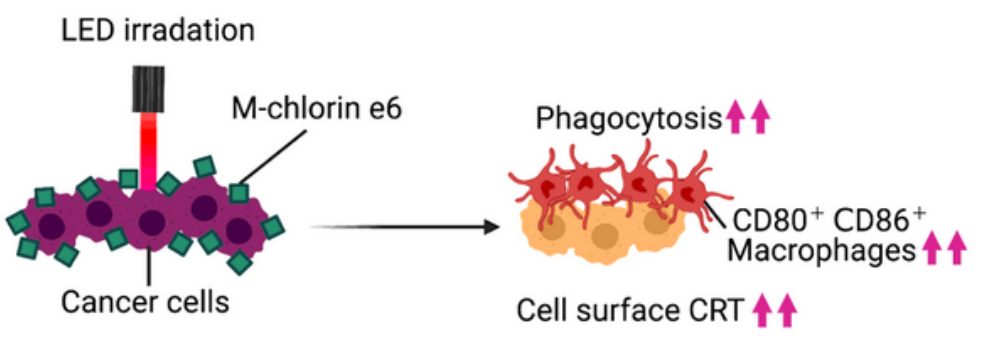

Figure 2

Effect of M-chlorin e6 PDT on the proportion of CD80+CD86+ macrophages and hypothetical anti-cancer mechanism of M-chlorin e6 PDT. (A) Representative results of flow cytometric analysis after $24 \mathrm{~h}$ of coculture with macrophages. (B) Proportion of CD80+CD86+ RAW264.7 cells. Data are presented as mean $\pm S E\left(n=3 ;{ }^{*} p<0.01\right.$; Student's t-test). (C) Summary of research results from the previous report. Mchlorin e6 PDT reduces the proportion of tumor-promoting M2-TAMs while increasing the proportion of 
tumor-suppressive M1-TAMs. M-chlorin e6 PDT also directly damages tumor cells. M-chlorin e6 PDT shrinks tumors via this combined mechanism. (D) Summary of research results from the present study. M-chlorin e6 PDT promotes phagocytosis of cancer cells by macrophages by increasing expression of CRT on the surface of cancer cells. In addition, treatment of cancer cells with M-chlorin e6 PDT increases the proportion of $\mathrm{CD} 80+\mathrm{CD} 86+$ macrophages and enhances antigen presentation. 